$>$ Alors que l'on connaissait depuis longtemps l'existence de la flore intestinale, la découverte du rôle du microbiote intestinal dans la santé et la maladie est perçue comme une des avancées scientifiques les plus importantes des dernières années. De nombreuses maladies chroniques résulteraient non pas de l'agression de microorganismes pathogènes, mais d'une rupture de l'équilibre de la population bactérienne présente dans l'intestin. Les dernières avancées dans ce domaine ont une portée qui dépasse largement les enjeux de santé publique que représentent le développement de régimes personnalisés ou d'alicaments et les perspectives de transplantation fécale à visée thérapeutique. Ces nouvelles connaissances modifient notre vision des microbes, et notre conception de la santé et de la maladie, du soi et du non-soi, les représentations du corps et des relations de l'individu avec son environnement. Le microbiote intestinal tend aujourd'hui à être considéré comme un organe à part entière, et l'organisme humain se définit comme un écosystème, un super-organisme chimérique possédant un double génome, humain et microbien. Une réflexion s'impose sur la façon dont ces nouveaux paradigmes viennent modifier les perceptions profanes sur le corps humain. <

Des milliards de microorganismes peuplent le corps humain et colonisent toutes nos interfaces corporelles. Ils sont partout: sur la peau, dans les poumons, dans la cavité vaginale, dans la bouche et la salive, dans la gorge, le rhinopharynx, et dans le système digestif. Leur nombre égale celui des cellules qui constituent l'organisme, aux environs de 10000 milliards [1]. C'est dans le tube digestif et plus particulièrement dans le gros intestin, qu'ils sont le plus nombreux; ils représentent 35 à $50 \%$ en volume du contenu intestinal, pour un

Vignette (Photo @ Inserm - Sébastien Petiot - CNDP/Universcience/MGEN/Inserm/ EDUCAGRI)

\section{Les enjeux médicaux et sociétaux du microbiote}

\section{Betty Dodet}

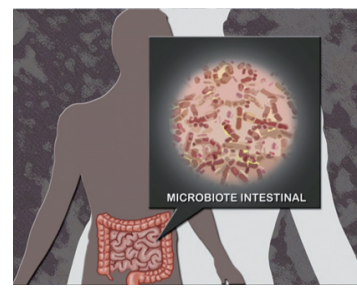

$6 \mathrm{~B}$, rue de Verdun, 69300 Caluire et Cuire, France. betty.dodet@dodetbioscience.com

poids de plus d'un kilogramme, et ils constituent ce que l'on appelle maintenant le microbiote intestinal.

L'étude du microbiote mobilise la communauté scientifique et les médias s'en font largement le relais auprès du grand public [35] $(\rightarrow)$. La découverte de l'impact des communautés microbiennes $(\rightarrow)$ Voir le Forum de C. Durif-Bruckert, page 1009 de ce numéro sur la santé humaine est largement perçue comme une des avancées importantes de ces dernières années.

\section{De la flore intestinale au microbiote : des rendez-vous manqués?}

II y a quelques années, le terme de microbiote était encore pratiquement inconnu, y compris des professionnels de la santé. Pourtant, on connaissait depuis longtemps son existence, sous un autre terme: la «flore intestinale » désignait les différentes populations bactériennes présentes naturellement dans l'intestin sans engendrer de pathologie, et dont on savait aussi qu'elles participaient au fonctionnement de l'organisme.

Dès 1885, Louis Pasteur proposait, dans une note à l'Académie des sciences, d'étudier le rôle des microbes dans la digestion, en maintenant des animaux dans un environnement stérile, « avec l'idée préconçue que la vie, dans ces conditions, deviendrait impossible» [2]. On peut alors se demander, «pourquoi chercheurs et médecins n'ont pris que très récemment conscience de l'importance du microbiote » ([3], p. 65). «Plus on revient sur les travaux qui témoignent d'une inflexion possible vers la notion de microbiote, plus on a l'impression d'une succession de rendez-vous manqués, d'évidences oubliées, de faits occultés » constate Patrice Debré ([3], p. 64) dans son livre L'homme microbiotique qui présente "les mille et une facettes de la vie en commun de l'homme et des microbes » ainsi que les différentes étapes de la « conquête du microbiote » [33] $(\rightarrow)$. En fait, Pasteur n'a pas pris le temps de mener les recherches proposées, préoccupé par ses travaux $\rightarrow$ Voir l'éditorial de P. Debré, page 919 de ce numéro 
sur les vaccins : c'est à cette époque que Joseph Meister a été vacciné contre la rage... L'intérêt des microbiologistes se concentre essentiellement, à ce moment, sur la lutte contre les maladies d'origine infectieuse, principale cause de mortalité. Ces recherches sont couronnées de succès avec l'identification de nombreux agents responsables de maladies $^{1}$ et, au $x x^{\mathrm{e}}$ siècle, avec la découverte des antibiotiques et le développement des vaccins. On en vient même à penser que les maladies infectieuses sont proches d'être vaincues et à rêver d'un monde sans microbes...

Le désintérêt pour l'étude de la flore intestinale s'explique également par les difficultés auxquelles se heurte son étude. II est en effet pratiquement impossible de cultiver les bactéries intestinales. Essentiellement anaérobies, elles meurent en présence d'oxygène. Même avec la mise au point de techniques de culture des bactéries anaérobies, dans les années 1960, la fraction d'espèces bactériennes accessibles reste encore limitée.

Tout va changer au début des années 2000 avec l'essor du séquençage à haut débit et des technologies dites «omiques», et avec l'augmentation des capacités informatiques de stockage et d'analyse des données. On peut dorénavant accéder à l'ensemble des gènes de la flore intestinale... sans avoir à passer par la culture bactérienne. Il est alors possible d'obtenir une vision globale des communautés microbiennes complexes qui habitent l'intestin, de les cataloguer et d'étudier comment l'ensemble des bactéries et leurs centaines de milliers de gènes coopèrent et interagissent avec l'organisme qui les héberge. La flore intestinale entre dans l'ère du Big Data et change d'appellation...

Parallèlement à ces développements, on s'intéresse de plus en plus aux maladies non transmissibles que sont le cancer ou les maladies cardiovasculaires, principales causes de décès dans les pays occidentaux. On se préoccupe de l'augmentation mondiale du nombre de personnes atteintes d'obésité et de diabète. Toutes ces maladies chroniques sont liées à l'environnement et au mode de vie, notamment à l'alimentation. L'étude du microbiote intestinal trouve donc sa place.

\section{De la flore intestinale au microbiote : des secrets révélés}

À la suite du programme de séquençage du génome humain (entre 1990 et 2003), de grands programmes d'étude du microbiome (fondée sur l'étude des gènes du microbiote) se mettent en place : Le Human Microbiome Project (HMP) américain [4] débute en 2007, le projet Metagenomics of the Human Intestinal Tract (MetaHIT) européen [5], en 2008. Ils ont permis en cinq ans de séquencer le métagénome de plusieurs centaines d'individus et d'étudier la structure, la fonction et la diversité des microbiomes humains. Ces études établissent un catalogue de plus de 3 millions de gènes microbiens - un catalogue 100 fois plus important que celui des gènes humains [6]. Le nombre d'espèces bactériennes identifiées au sein de l'ensemble des métagénomes

${ }^{1}$ Identification des agents responsables de la lèpre (Hansen, 1873), de la gangrène gazeuse (Pasteur, 1877), de la typhoïde (Eberth, 1880), de la tuberculose (1882) et du choléra (1883) par Robert Koch, de la diphtérie (Loeffler, 1884), du tétanos (Nicolaier, 1884), de la peste (Yersin, 1894), des souches pathogènes d'Escherichia coli (Escherich, 1885) pour n'en citer que quelques-uns. humains analysés se situerait entre 1000 et $1200[4,7$, 8]. Il est ainsi possible de répartir la population bactérienne en trois groupes ou entérotypes selon la prépondérance de certains groupes de bactéries intestinales, chaque individu hébergeant une composition bactérienne intestinale d'environ 160 espèces différentes qui lui est propre, tout en partageant un noyau commun constitué d'une soixantaine d'espèces dominantes [7, 8]. Le microbiote intestinal semble avoir les propriétés d'un organe à part entière: situé à l'interface de l'organisme et de son environnement. II a une morphologie, une physiologie et une physiopathologie qui lui sont propres. Mais à la différence des autres organes, il ne se forme qu'après la naissance. Stérile in utero, le tube digestif du nouveau-né est colonisé dès les premières heures de la vie par les germes de sa mère et de son environnement. II se diversifie progressivement au moment du sevrage et de l'introduction d'aliments solides pour se stabiliser vers l'âge de deux à trois ans. La composition du microbiote est donc déterminée par un ensemble de facteurs génétiques et environnementaux.

On découvre que le microbiote intestinal a un rôle essentiel dans le fonctionnement de l'organisme. Les bactéries qui le constituent dégradent les composés d'origine alimentaire et les transforment en nutriments énergétiques; elles contrôlent le métabolisme des sucres et des lipides, le métabolisme osseux, la réparation épithéliale, la maturation des vaisseaux ; elles synthétisent des vitamines, des enzymes, des neurotransmetteurs. Elles contribuent à la maturation du tube digestif, jouent un rôle de barrière contre les microorganismes pathogènes, neutralisent de nombreuses substances toxiques. Le microbiote participe au développement et à la maturation du système immunitaire et du système nerveux central

$(\rightarrow)$ Voir la Nouvelle de S. Normand et al., $m / s n^{\circ} 6-7$, juinjuillet 2013, page 586 et pourrait moduler le comportement $[9,10](\rightarrow)$. Si ces populations bactériennes jouent un rôle central dans le fonctionnement de l'organisme, de nombreuses études suggèrent qu'une rupture d'équilibre de cette symbiose est impliquée dans des maladies aussi variées que les maladies inflammatoires de l'intestin [36] $(\rightarrow)$, les maladies métaboliques [11] $(\rightarrow)$, l'ostéoporose [12], les allergies, les cancers [13] $(\rightarrow)$ et même les troubles du comportement alimentaire et les troubles psychiques comme l'autisme ou la dépression, ou encore la sclérose $(\rightarrow)$ Voir la Synthèse de R. Burcelin et al., $m / s n^{\circ} 8-9$, aoûtseptembre 2013, page 800

$(\rightarrow)$ Voir la Synthèse de 0 . Rahmouni et al., page 968 de ce numéro

$(\rightarrow)$ Voir la Synthèse de J. Raisch et al., $\mathrm{m} / \mathrm{s}$ $n^{\circ} 2$, février 2016, page 175 
en plaques. Beaucoup d'éléments à ce sujet demeurent inexpliqués et nécessitent d'explorer les interactions complexes entre les différentes populations microbiennes avec leur hôte et leur environnement. II est reconnu qu'une grande diversité bactérienne au sein du microbiote, serait l'expression d'un microbiote sain (l'«eubiose »). Une dysbiose, qui se caractérise par une diversité plus faible et la prédominance de certaines bactéries, se révèle dans de très nombreuses maladies $[9,14](\rightarrow)$. Des maladies chroniques qualifiées de postmodernes telles que l'obésité et le diabète sont $(\rightarrow)$ Voir la Nouvelle de S. Normand et al., $m / s n^{\circ} 6-7$, juinjuillet 2013, page 586 associées à des compositions bactériennes particulières. Elles se caractérisent par une diminution de la diversité bactérienne et une modification de l'abondance relative de différentes espèces de bactéries [15]. Ainsi, le transfert du microbiote de souris obèses à des souris minces rend ces dernières obèses à leur tour [16]. On envisage de prévenir ou de traiter toutes ces pathologies liées à la dysbiose en modifiant le microbiote intestinal, par des régimes personnalisés, des probiotiques et des prébiotiques, ou même en manipulant le microbiote par des transplantations $[11,17,18,34](\rightarrow)$.

La liste des maladies susceptibles de bénéficier de tels traitements ne cesse de s'allonger, au fur $(\rightarrow)$ Voir la Synthèse de R. Burcelin et al., $\mathrm{m} / \mathrm{s} \mathrm{n}{ }^{\circ} 8$-9, aoûtseptembre 2013, page 800 , et la Synthèse de J.C. Lagier et D. Raoult, page 991 de ce numéro et à mesure de la découverte de nouvelles fonctionnalités du microbiote. On peut se demander s'il agit d'un mythe, ou d'une réalité. Qu'est ce qui est faisable? Qu'est-ce qui est acceptable ?

\section{Mythe ou réalité ?}

Pour l'instant, les applications à visée préventive ou thérapeutique sont très limitées et s'inscrivent dans des approches que l'on peut considérer comme «classiques ». Néanmoins, ces découvertes biomédicales ont des répercussions individuelles et sociales qu'il convient d'accompagner et d'anticiper. Les sciences humaines et sociales sont ainsi concernées tout autant que les sciences biomédicales. C'est dans ce contexte qu'a eu lieu à Lyon un «Atelier microbiote», le premier d'une série de rencontres interdisciplinaires sur les « Enjeux contemporains en santé $»^{3}$. Cet atelier visait à mettre en relation, autour des questions liées au microbiote, la communauté des chercheurs de différentes disciplines avec les acteurs clés concernés, et à initier une réflexion commune sur les retombées biomédicales, sociales et économiques de ces découvertes scientifiques.

\section{Vers des régimes alimentaires personnalisés?}

On connaît déjà, indépendamment du microbiote, des régimes alimentaires qui favorisent l'obésité, le diabète ou certains cancers. La connaissance du microbiote particulier qu'héberge un individu pourrait aider à affiner le régime lui convenant le mieux. Des recherches

\footnotetext{
${ }^{2}$ On peut légitimement se demander ce qui est faisable ou acceptable en termes de traitement.

${ }^{3}$ Atelier microbiote SHS - Lyon, mars 2015, organisé par des chercheurs des Universités Lyon 2 et Lyon 3, Mérieux Université et la Fondation pour l'Université de Lyon.
}

se poursuivent dans l'espoir d'établir les bases d'une nutrition préventive et curative qui serait adaptée à chaque individu ou groupe d'individus, pour la production d'aliments dits fonctionnels, de prébiotiques et de probiotiques capables de maintenir ou de rétablir l'équilibre du microbiote.

Plusieurs grands programmes, comme le Hundred Person Wellness Project (HPWP) aux États-Unis [19], Metagenopolis ${ }^{4}$ [20] ou My.microbes [21] en Europe, visent à collecter les données individuelles sur le microbiome ou le métagénome issues de l'analyse réalisée chez des milliers de volontaires, dans la perspective du développement d'une médecine «préventive et personnalisée » [22] $(\rightarrow)$.

$(\rightarrow)$ Voir le Forum de M. Billaud et $X$. Guchet, $m / s n^{\circ} 8-9$, août-septembre 2015, page 803 La modulation du microbiote intestinal représente un nouveau paradigme, en termes de politique nutritionnelle et de santé publique, suggérant un investissement collectif autant qu'un investissement personnel du citoyen-consommateur. Ces nouvelles approches de la santé, de la maladie et des traitements appellent à un vaste débat impliquant la société tout entière.

On voit déjà se développer un marché des compléments alimentaires et des aliments de santé ou «aliments fonctionnels ». Ces développements représentent des enjeux considérables en termes économiques et sociaux. Ils soulèvent également des questions éthiques, sociales et réglementaires. Les autorités chargées de la réglementation comme l'EFSA (European Food Safety Authority) en Europe ou la FDA (Food and Drug Administration) aux États-Unis se penchent sur la question, alors que l'industrie alimentaire demande des directives sur les études à effectuer. Mais comment apporter la preuve que ces «alicaments», à la frontière entre aliments et médicaments, permettent de vivre en bonne santé ? Quels critères d'évaluation utiliser? Il reste à identifier des biomarqueurs permettant de montrer un effet favorable de certains aliments particuliers, de certains produits pré- et probiotiques sur le microbiote et, par conséquent, la santé.

\section{Un organe transplantable}

Considéré maintenant comme un organe à part entière, le microbiote intestinal peut être transplanté [34] $(\rightarrow)$.

\section{$(\rightarrow)$ Voir la Synthèse de J.C. Lagier et D. Raoult, page 991 de ce numéro} Pour l'instant, la transplantation de microbiote fécal s'est avérée efficace dans le traitement des infections

\footnotetext{
${ }^{4}$ Coordonné et hébergé par l'INRA à Lyon, MetaGénoPolis (MGP) a débuté en 2013 et rassemble 4 plateformes de recherches.
} 
intestinales récidivantes à Clostridium difficile [17], infections le plus souvent consécutives à une antibiothérapie entraînant une altération importante du microbiote intestinal. Plusieurs centaines de patients ont été traités ainsi avec succès. L'introduction des selles d'un donneur sain dans leur tube digestif a permis de restaurer leur flore intestinale altérée. Cette approche qui paraît novatrice a été publiée pour la première fois en 1958 (avant la « découverte » du microbiote), mais elle s'inscrit dans une longue histoire revisitée : le transfert de selles se pratiquait en Chine dès le Iv siècle [23]. Cette pratique pose néanmoins des questions quant à son acceptabilité. Quelle en est la perception de la population, des donneurs et des receveurs potentiels de matériel fécal $[24,35]$ ? $(\rightarrow)$. $\rightarrow$ Voir le Forum de C. Durif-Bruckert, page 1009 de ce numéro L'essor de la transplantation fécale pose en outre la question de l'encadrement et du statut juridique du transplant, sur lesquels les avis sont divisés $[25,26]$. S'agit-il d'un tissu au même titre que le cartilage par exemple, ou bien d'un médicament? Par ailleurs, les selles ne sont-elles qu'un substrat dans lequel prospère le microbiote? L'Agence européenne des médicaments ne s'est pas encore prononcée. À ce jour, en France, le code de la Santé publique ne prévoit pas de statut particulier, mais pour l'Agence de sécurité du médicament et des produits de santé (ANSM), le microbiote fécal utilisé en transplantation doit être considéré comme un médicament dans la mesure où il a une visée curative [27]. Le donneur apparaît alors comme une sorte de bioréacteur produisant un matériel d'intérêt thérapeutique [26]. Pour unifier et sécuriser la transplantation de microbiote fécal, qui entre déjà dans la pratique pour les infections récurrentes à Clostridium difficile, le Groupe français de transplantation fécale (GFTF) vient de publier des recommandations concernant ses indications, la sélection des donneurs, la préparation et l'administration du transplant, ainsi que sa traçabilité [28].

La même question se pose pour le don de matières fécales dans un but de recherche. Il s'avère en effet plus difficile de recueillir des échantillons de selles que des échantillons de sang, remarquent les chercheurs engagés sur le terrain, reconnaissant que l'on touche là à des aspects symboliques fondamentaux [35] $(\rightarrow)$. La constitution de banques de microbiote fécal doit donc s'accompagner d'une $(\rightarrow)$ Voir le Forum de C. Durif-Bruckert, page 1009 de ce numéro réflexion sur leur acceptabilité et de règles en matière de propriété, de confidentialité et d'utilisation des données. Dans cet esprit, MetaGenoPolis a créé une plateforme de vigilance éthique pour encadrer le « don de selles » et pour évaluer l'impact éthique et sociétal des projets de recherche en métagénomique dans le domaine de la santé, de la nutrition et de la médecine.

\section{De nouveaux paradigmes}

La révolution engendrée par les découvertes sur le microbiote n'est peut-être pas tant, actuellement, dans le domaine thérapeutique que dans la remise en question des paradigmes sur la santé et la maladie, des représentations du corps humain et de la place qu'il occupe dans son environnement.
Un nouveau regard sur les microbes, la maladie et la notion de norme

Alors que le terme de microbe a été indiscutablement associé à la notion de risque pathogène, il faut maintenant admettre que les bactéries pathogènes ne représentent qu'une faible fraction de la population bactérienne avec laquelle nous interagissons, la plupart jouant un rôle bénéfique pour notre santé.

Un «nouveau » concept de la santé et de la maladie se fait jour. Maintenant se développe la notion de maladies locales ou systémiques dues à la rupture de l'équilibre de l'écosystème bactérien présent dans le corps humain. En contraste, l'eubiose qualifierait l'état d'un microbiote intestinal dit équilibré, remplissant toutes les fonctions garantissant à son hôte santé et bienêtre. On sort du dualisme santé-maladie pour concevoir un continuum entre un équilibre considéré comme «parfait» et induisant un état de bien-être et de satisfaction, et les dysfonctionnements plus ou moins importants responsables de malaises et de maladies. On retrouve là les théories d'Hippocrate; les bactéries remplacent les humeurs.

Mais les limites entre l'eubiose et la dysbiose restent floues et la répartition entre «bonnes bactéries » et «mauvaises bactéries » n'a rien d'évident. L'équilibre tient-il à la diversité de la composition du microbiote, à la présence ou à la prédominance de certaines espèces bactériennes?

C'est donc la notion même de norme qui est ici remise en question. On peut s'interroger sur la définition d'un «bon microbiote», d'un microbiote encore dit normal ou d'un microbiote optimal, sachant que chaque individu possède un microbiote qui lui est spécifique au point de pouvoir être considéré comme une carte d'identité biologique. Dans ces conditions, la question centrale «comment moduler la dysbiose? »n'a pas une réponse unique et définitive. De même, la notion de «bons aliments », qui favoriseraient cet état d'eubiose reste à définir, tant du point de vue qualitatif que quantitatif, et la frontière entre aliment et médicament s'estompe. Les «bons aliments» sont-ils les mêmes pour tous, ou varient-ils en fonction des particularités de chaque individu et de son milieu?

\section{Le corps humain dans son écosystème}

À cet organe supplémentaire qu'est le microbiote intestinal, viennent s'associer les flores buccale, génitale, pulmonaire, cutanée, ce qui conduit à la notion de superorganisme, écosystème complexe tendant à faire un tout cohérent de l'homme et de ses microbes [29]. Ils ont évolué ensemble sur plusieurs dizaines de milliers d'années et établi au cours de leur évolution des relations 
mutualistes constituant une véritable symbiose ${ }^{5}$ [11] $(\rightarrow)$.

Cette symbiose estompe les limites entre intérieur et extérieur. Jusqu'ici, l'organisme humain était perçu comme clairement défini par son propre $(\rightarrow)$ Voir la Synthèse $\mathrm{m} / \mathrm{s} \mathrm{n}^{\circ} 8-9$, aoûtseptembre 2013 page 800 de R. Burcelin et al. génome, et son système immunitaire était chargé d'éliminer tout ce qui lui était étranger, virus, bactéries, ou tissus provenant d'un donneur non apparenté. L'étude du microbiote nous révèle aujourd'hui que l'homme ne se définit pas seulement par ses gènes et ses cellules, mais par l'ensemble qu'il forme avec les microbes qu'il abrite. Ce point rouvre le débat sur la définition du soi et du non-soi biologique; les bactéries commensales enseignent que l'autre peut être un constituant majeur et indispensable du soi.

\section{L'émergence de «l'homme microbiotique 》}

Les nouvelles connaissances sur le microbiote amènent à redéfinir l'individu biologique, avec l'avènement de nouveaux modèles du corps qui devraient induire de nouvelles représentations. Ces représentations du corps physiologique ont à plusieurs reprises évolué au cours des siècles, en fonction des connaissances scientifiques et de l'avancement des technologies, le passage d'un modèle représentatif à un autre ne conduisant pas forcément à la disparition des modèles antérieurs.

Pour beaucoup d'historiens, la notion de corps «moderne», objet de la médecine et de l'anatomie, naît en 1543, dans les planches du traité d'anatomie de Vésale [30]. Au xvII siècle, Descartes (15961650) propose le modèle mécanique d'une machine, d'un automate, comparable à une horloge composée de roues et de contrepoids, ou à un moulin que le cours ordinaire de l'eau peut rendre continu [31, 32]. Au xIx $\mathrm{x}^{\mathrm{e}}$ siècle, avec l'avènement de l'ère industrielle, le corps est plutôt assimilé à un moteur produisant de l'énergie. Dans la deuxième partie du $x x^{e}$ siècle, avec le développement de l'informatique, le décryptage du code génétique suggère le modèle de l'ordinateur. La structure et le fonctionnement du corps humain pourraient se déduire de son programme génétique, explication proposée pour de nombreuses maladies à composante génétique que l'on espère guérir en remplaçant les gènes mutés ou absents.

Au moment où se manifeste la préoccupation des grands équilibres écologiques planétaires et grâce au développement de la métagénomique, s'élabore maintenant le modèle de «l'homme microbiotique», ainsi que le nomme Patrice Debré $[3,33](\rightarrow)$.

$\rightarrow$ Voir l'éditorial de P. Debré, page 919 de ce numéro Un nouveau modèle du corps s'ébauche. Le corps lui-même est présenté comme un écosystème, un super-organisme chimérique possédant un double génome, humain et microbien, dont les fonctions seraient complémentaires.

Une réflexion s'impose sur la façon dont ces nouveaux paradigmes et ces nouvelles représentations viennent modifier les perceptions profanes sur le corps humain. $\diamond$

${ }^{5}$ Association entre deux ou plusieurs organismes profitable à chacun d'eux.

\section{SUMMARY}

Microbiota and representations of the human body Although the presence of an intestinal flora has been known for a long time, the discovery of the role of gut microbiota in human health and disease has been widely recognized as one of the most important advances in the recent years. Chronic diseases may result from dysbiosis, i.e. a disruption of the balance within the bacterial population hosted by the human body. These developments open new prospects in terms of prevention and treatment, including the design of adapted diets, the development of functional foods and fecal transplantation. These discoveries have profoundly altered our view of microbes, of health and disease, of self and non-self, as well as our representations of the body and its relationship with its ecosystem. Gut microbiota is now generally considered as an organ in its own right. A model of the "microbiotic person" thus arises, in which the human organism is defined as an ecosystem, a chimeric superorganism with a double genome, both human and microbial. Thought should be given to the way in which these new paradigms modify lay perceptions of the human body. $\diamond$

\section{REMERCIEMENTS}

Nous remercions particulièrement Élodie Giroux, Christine DurifBruckert, Catherine Dekeuwer, Vincent Baty et Bruno Mougin, organisateurs de l'Atelier Microbiote, ainsi que l'ensemble des intervenants et participants de cet Atelier.

\section{LIENS D'INTÉRÊT}

L'auteur déclare n'avoir aucun lien d'intérêt concernant les données publiées dans cet article.

\section{RÉFÉRENCES}

1. Sender R, Fuchs $S$, Milo R. Are we really vastly outnumbered? revisiting the ratio of bacterial to host cells in humans. Cell $2016 ; 164: 337-40$.

2. Pasteur L. Observation relative à la note précédente de M. Desclaux. Comptes Rendus de l'Académie des Sciences $1885 ; 100$.

3. Debré P. L'homme microbiotique. Paris : Odile Jacob, 2015 : 288 p.

4. Human Microbiome Project Consortium. Structure, function and diversity of the healthy human microbiome. Nature $2012 ; 486: 207-14$.

5. MetaHit. http://www.metahit.eu/

6. Shreiner AB, Kao JY, Young VB. The gut microbiome in health and in disease. Curr Opin Gastroenterol $2015 ; 31: 69-75$.

7. (in J, Li R, Raes J, et al. A human gut microbial gene catalogue established by metagenomic sequencing. Nature $2010 ; 464: 59-65$.

8. Arumugam M, Raes J, Pelletier $\varepsilon$, et al. Enterotypes of the human gut microbiome. Nature $2011 ; 473: 174-80$.

9. Normand $S$, Secher, T, Chamaillard M. La dysbiose, une nouvelle entité en médecine? Med Sci (Paris) $2013 ; 29: 586-9$.

10. Erny D, de Angelis AL, Prinz M. Communicating systems in the body: how microbiota and microglia cooperate. Immunology 2016, 24 August. doi: 10.1111/imm. 12645

11. Burcellin R, Chabo C, Blasco-Baque V, et al. Le microbiote intestinal à l'origine de nouvelles perspectives thérapeutiques pour les maladies métaboliques? Med Sci (Paris) 2013 ; $29: 800-6$.

12. Weaver CM. Diet, gut microbiome, and bone health. Curr Osteoporos Rep $2015 ; 13: 125-30$. 


\section{RéFÉRENCES}

13. Raisch J, Dalmasso G, Bonnet R, et al. Certaines bactéries de la flore commensale exacerberaientelles la carcinogenèse colorectale? Med Sci (Paris) 2016 ; 32 : 175-82.

14. Kahlert C, Müller P. Microbiome, découverte d'un organe. Forum Med Suisse 2014 ; 14 : 342-4.

15. Baothman OA, Zamzami MA, Taher I, et al. The role of gut microbiota in the development of obesity and diabetes. Lipids Health Dis 2016 ; 15 : 108.

16. Turnbaugh PJ, Ley RE, Mahowald MA, et al. An obesity-associated gut microbiome with increased capacity for energy harvest. Nature $2006 ; 444: 1027-31$.

17. Gupta S, Allen-Vercoe $\varepsilon$, Petrof $\varepsilon 0$. Fecal microbiota transplantation: in perspective. Therap Adv Gastroenterol $2016 ; 9: 229-39$.

18. McCarville JL, Caminero A, Verdu EF. Novel perspectives on therapeutic modulation of the gut microbiota. Therap Adv Gastroenterol $2016 ; 9: 580-93$.

19. Institute for Systems Biology. 100K Wellness Project. http://research.systemsbiology.net/100k/

20. Metagenopolis. the microbiota in human health and well being. http://www.mgps.eu/index. php?id=accueil\&L=1

21. My.Microbes. http://my.microbes.eu/

22. Billaud M, Guchet X. L'invention de la médecine personnalisée. Med Sci (Paris) 2015 ; 31 : 797-803.

23. Zhang F, Luo W, Shi Y, et al. Should we standardize the 1,700-year-old fecal microbiota transplantation? Am J Gastroenterol $2012 ; 107: 1755$.

24. Kahn SA, Gorawara-Bhat R, Rubin DT. Fecal bacteriotherapy for ulcerative colitis: patients are ready, are we? Inflamm Bowel Dis 2012 ; 18 : 676-84.

25. Smith MB, Kelly C, Alm $\varepsilon$ J. Policy: how to regulate faecal transplants. Nature $2014 ; 506: 290-1$.

26. Megerlin F, Fouassier $\varepsilon$, Lopert $R$, Bourlioux P. Faecal microbiota transplantation: a sui generis biological drug, not a tissue. Ann Pharm Fr $2014 ; 72$ : 217-20.

27. ANSM. La transplantation de microbiote fécal et son encadrement dans les essais cliniques. Agence nationale de sécurité du médicament et des produits de santé, 2014. http://ansm.sante. $\mathrm{fr} / v a r / a n s m \_s i t e / s t o r a g e / o r i g i n a l / a p p l i c a t i o n / 5 e 5 e 01018303790194275 \mathrm{ded0} 002353 \mathrm{c} . p d f$

28. Sokol H, Galperine T, Kapel N, et al. Faecal microbiota transplantation in recurrent Clostridium difficile infection: recommendations from the French group of faecal microbiota transplantation. Dig Liver Dis $2016 ; 48: 242-7$.

29. Sansonetti P. La relation hôte-microbiote : une insondable symbiose ? Lettre du Collège de France $2011 ; 32$.

30. Benoist J, Cathebras P. The body: from an immateriality to another (1993). Conception et représentations du corps. Soc Sci Med 1993 ; $36: 857-65$.

31. Descartes R. Traité de l'Homme. Leyde, 1662

32. Legée G. L'Homme de René Descartes (éditions de 1662 et 1664) : physiologie et mécanisme. Communication présentée à la séance du 19 décembre 1987 de la Société française d'Histoire de la Médecine. Histoire des sciences médicales. Paris, 1987, tome XXI, n ${ }^{\circ}$, pp. 365-80.

33. Debré P. Les défis du microbiote. Med Sci (Paris) 2016 ; 32 : 919-20.

34. Lagier JC, Raoult D. Greffe de microbiote fécal et infections : mise au point, perspectives. Med Sci (Paris) $2016 ; 32: 991-7$.
35. Durif-Bruckert C. Microbiote intestinal et émergence de nouvelles représentations du corps : une approche psychosociale. Med Sci (Paris) 2016 ; 32 : 1009-15.

36. Rahmouni 0 , Dubuquoy L, Desreumaux P, Neut C. Microbiote intestinal et développement des maladies inflammatoires chroniques de l'intestin. Med Sci (Paris) $2016 ; 32: 968-73$.

\section{OUVRAGES DE RÉFÉRENCE}

- Patrice Debré. L'homme microbiotique. Paris : Odile Jacob, 2015 : 288 pages.

- Philippe Sansonetti. Cours et séminaires au Collège de France. http://www. college-de-france.fr/site/philippe-sansonetti/_course.htm

- Philippe Sansonetti. Leçon inaugurale 2008-2009. Des microbes et des hommes. Guerre et paix aux surfaces muqueuses. http://www.college-defrance.fr/site/philippe-sansonetti/_inaugural-lecture.htm

- Alain Corbin, Jean-Jacques Courtine, Georges Vigarello (coordination). Histoire du corps, 3 vol. Collection L'Univers historique. Paris : Seuil, 20052006.

POUR EN SAVOIR PLUS

- Barbut F, Joly F. Le microbiote intestinal : équilibre et dysbiose. Hep Gastro Oncol Digest $2010 ; 17 ; 511-20$.

- Baty V, Mougin B, Dekeuwer C, Carret G. Gut health in the era of the human gut microbiota: from metaphor to biovalue. Med Health Care Philos 2014 ; 17 : 579-97.

- Dore J, Simren M, Buttle L, Guarner F. Hot topics in gut microbiota. UEG Journal $2013 ; 1$ : 311-8.

- Gilbert JA, Neufeld JD. Life in a world without microbes. PLoS Biol 2014 ; 12 : el002020.

- Inra. Microbiote intestinal, nouvel organe au potentiel extraordinaires. http://www.inra.fr/Grand-public/Alimentation-et-sante/Tous-lesdossiers/Metagenome-intestinal

- Gut Microbiota World Watch, diverses ressources du Centre d'Information sur le microbiote intestinal proposé par la Société Européenne de Neurogastroentérologie et de Motilité sur http://www.gutmicrobiotawatch. org/fr/

- Microbiote : des bactéries qui nous veulent du bien. CNRS - Le Journal. https://lejournal.cnrs.fr/articles/microbiote-des-bacteries-qui-nousveulent-du-bien

- Groenen H. Une histoire des représentations du corps, 2006-2007. campusport.univ-lille2.fr/doc-lic_1-4/hist_corps_haimo.pdf

\section{TIRÉS À PART}

B. Dodet

\section{Bon de commande}

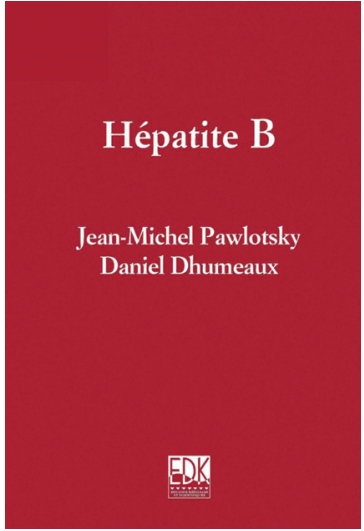

ISBN : 978-2-8425-4131-6 576 pages
À retourner à EDK, 109, avenue Aristide Briand, 92541 Montrouge Cedex Tél. : 0141177405 - Fax : 0149850345 - E-mail : francois.flori@edpsciences.org NOM : Prénom :

Adresse :

Code postal :

Ville :

Pays :

Fonction :

Je souhaite recevoir l'ouvrage Hépatite B : $54 €+3 €$ de port $=\mathbf{5 7} €$ TTC offre exceptionnelle réservée aux abonnés à $\mathrm{m} / \mathrm{s}$ jusqu'au 31 décembre 2010

en ................ exemplaire, soit un total de ..................................... €

$\square$ Par chèque, à l'ordre de EDP Sciences

$\square$ Par carte bancaire : $\square$ Visa $\square$ Eurocard/Mastercard

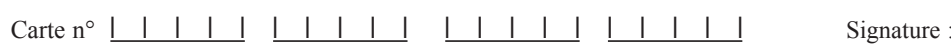

Date d'expiration: $\quad$ । 11111

$\mathrm{N}^{\circ}$ de contrôle au dos de la carte : 